\title{
Improving Internet Coverage in Rural Africa by Using Passive Repeaters in the Home
}

\author{
P. Lynggaard \\ CMI, Aalborg University Copenhagen Copenhagen, Denmark \\ E-mail:perlyn@es.aau.dk \\ Received 16 June 2015; \\ Accepted 17 December 2015
}

\begin{abstract}
Providing last-mile Internet access to rural areas in Africa is a very challenging task, i.e. many more or less successful attempts have been made over the years. One solution that seems to offer a sustainable business case is based on WiFi base stations with access points placed in the centers of the villages. Nevertheless, this solution has a limited range, especially inside the homes where the wall attenuation dominates. This paper provides a solution in the form of deploying a cheap, simple, and powerless passive repeater on the shielding walls. By simulating the WiFi radio-link between a base station and a user positioned inside a home, it has been found that the passive repeater lowers the wall attenuation considerably and thereby extends the usable range.
\end{abstract}

\section{Introduction}

Internet technologies for rural areas need to deal with challenges, limitations and shortcomings such as lack of power, lack of money, and lack of infrastructure. A first choice solution could be to use "fixed" Internet connections in the form of cables and optical fibers, but this solution is costly and it requires that the residents are physically located close to the exchange points [1]. Thus, in many rural areas and developing countries the Internet Service Providers (ISP) need to invest in infrastructure such as long optical cables, repeaters,

Journal of NBICT, Vol. 1, 65-80.

doi: 10.13052/NBICT.2016.004

(C) 2016 River Publishers. All rights reserved. 
main switches, exchange switches, and other components. This means that the exchange switches must be positioned in densely populated areas, i.e. medium size towns, etc. to achieve a high return of investment rate, but in many rural areas the population is limited and the income is low, i.e. the digital divide dominates. An alternative to the wired connections are wireless solutions for rural areas [2]. One of the promising candidates is a concept provided by a Danish company named "BlueTown". Their solution is based on WiFi and offers low cost, full network integration, and low form factor WiFi base station towers. Technically, these towers offer multiple access points, they are solar powered, and they are equipped with rechargeable batteries. Placing these towers in the centers of the villages means that many users can share these towers simultaneously. Nevertheless, this solution has some disadvantages in the form of the limited range the WiFi standards (802.11x) offer and in the form of the limited transmission power available in the mobile phone return path. This is a major problem when the users want to access the Internet from inside their homes where the WiFi return signal is damped considerably by the walls. This challenge could be overcome by providing external antennas connected to the mobile phones with cables. However, most cheap mobile phones do not have an external antenna input and such solutions also limit the flexibility of the portable devices.

A more beneficial solution is to provide a passive repeater, which consists of two connected passive antennas. One antenna is placed outside the home pointing at the WiFi base station and the other antenna is placed inside the home. These antennas are connected by a cheap electronic phase shifter and they can be designed as flat micro-strip-arrays that are easy to mount and hide on the walls.

This paper focuses on indoor WiFi coverage in the BlueTown solution and it discusses the achievements that can be gained by deploying passive repeaters in a small African village. Indoor WiFi coverage is central for supporting indoor Internet services which influences the financial growth and the social well-beings for people [3]. In contrast, mobile phone voice calls are normally handled in the current context which can be both indoor and outdoor.

The methodology used is deriving the needed formulas for connecting a mobile phone to a BlueTown access point as a function of distance, wall attenuation, and by using a passive repeater solution. These formulas are simulated in a mathematical tool and the elaborated results are presented and discussed. It has been found that indoor coverage is a problem even a few hundred meters from the WiFi base stations and the attenuation from the 
house walls considerably reduces this distance. However, the usage of a simple cheap passive repeater to some extent outweighs the wall losses and thereby increases the indoor coverage, i.e. the availability of the Internet from inside the home.

\section{Related Works}

Many different solutions have been deployed to deal with the challenges of providing Internet to rural areas. Matthee et al. [3] discuss the work done by the LinkNet organization to provide Internet to a small province in the southern of Zambia. Their solution uses a satellite based terminal as the main node, which can be accessed from the users through a wired network. However, this solution requires an up-front investment of $120.000 \$$ and $108.000 \$$ in maintenance costs over 10 years for a shared bandwidth of 1.024 Mbps (downlink) per satellite terminal. The authors conclude that the cost is one of the most important issues that must be solved.

Similarly, a work performed by Mardeni et al. [4] discusses a concept based on Wireless Regional Access Networks (WRAN). They compare a WRAN based solution in Zimbabwe with other technologies such as WiMAX and mobile cellular with respect to bandwidth and the number of subscribers. They found that a WRAN solution provides more bandwidth at a cheaper price than solutions based on WiMAX and cellular concepts. However, they also foresee problems with interferences from the existing TV stations that own and utilize the spectrums. Additionally, the WRAN concept has problems in the form of "hidden nodes".

A low cost solution that provides last-mile access using the unlicensed band of $2.4 \mathrm{GHz}$ in combination with existing infrastructure is provided by BlueTown. Their solution offers public access points through managed hotspots. Basically, their solution provides WiFi access points at low costs, high integration, and low power consumption. The core technology is small solar powered towers, which contain the routers, controllers, and access points. These towers support a backhaul bandwidth of $10 \mathrm{Mbps}$ in a circular range of $1 \mathrm{~km}$ with a tower height of $25 \mathrm{~m}$ [5]. The advantage in this solution is the possibility to use cheap handsets in the form of low cost mobile phones with or without a SIM card and furthermore, the use of existing infrastructure in the form of partnership with the existing ISP's.

The technology of passive repeaters together with the basic modeling concept used in this work has been discussed in [6]. 


\section{A Technology based Solution}

In this section the challenge of indoor coverage in the BlueTown solution is discussed, together with a passive repeater device, which is modeled, simulated, and elaborated.

\subsection{The BlueTown Solution}

The BlueTown solution is based on placing WiFi access points in the core of the sparsely populated villages in the rural part of Africa, which means they are reachable for most of the nearby citizens [7, 8] as illustrated in Figure 1.

The BlueTown solution provides Internet access by connecting to the fibers or wireless micro-links provided by the existing Internet backhaul, which is offered by the local service and infrastructure providers. These microoperators are positioned in the rural villages, where they deploy Internet connections in the form of WiFi base-stations. The end-user then connects to the access-point offered by the base-stations by using WiFi enabled devices like, e.g. mobile phones (smart phones).

This solution provides WiFi access in a circular Line Of Sight (LOS) range limited to approximately 1 kilometer. The primary limiting factor is the acknowledgement scheme used by the WiFi (IEEE 802.11x) standards and transmission power available in the mobile phones. The limits set by the WiFi acknowledgement scheme restrict the range to approximately $1.6 \mathrm{~km}$,

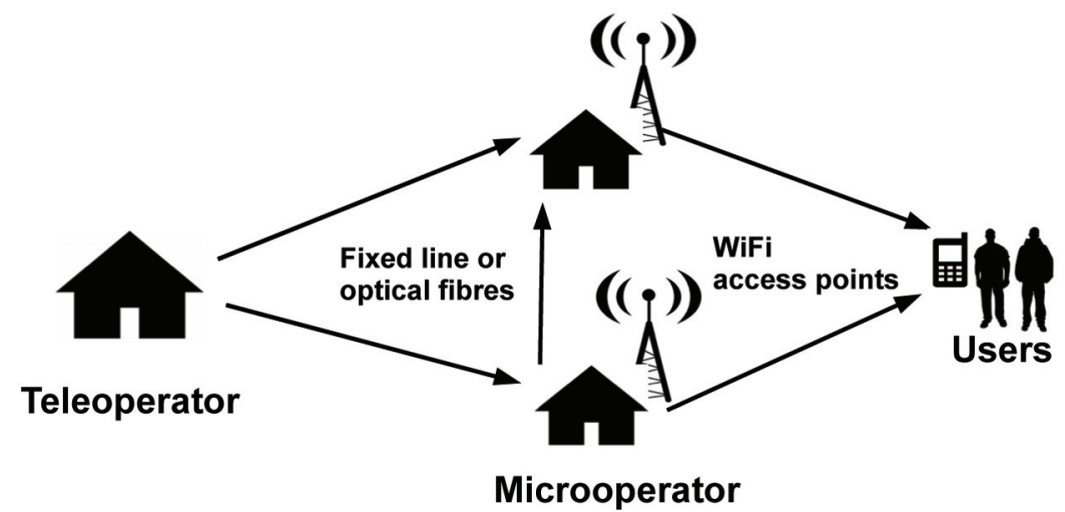

Figure 1 The BlueTown solution. A Teleoperator located in an Internet covered city provides Internet to the micro-operators by using fixed lines, radio link and optical fibers. The microoperators deploy WiFi base-stations with access points to distribute Internet access to the end-users. 
because each transmitted frame must be acknowledged before a the lost frame timeout expires. Thus, the travel-time for the radio-waves must be below a certain limit to avoid triggering a re-transmission from the sender, because it did not receive the acknowledgement frame in time. Likewise, many of the protocols used in accessing the services on the Internet also have similar acknowledge requirements. Regarding the WiFi transmission power used by the mobile phones it is often limited to save battery lifetime. This limitation provides some challenges in the form of overcoming limiting factors such as background noise, interferences, poor antennas without Multiple Input and Multiple Output (MIMO), and the usage of high frequencies $(2.4 \mathrm{GHz}$ or $5 \mathrm{GHz}$ ). The offered WiFi transmission power is set by the different equipment manufacturers, but in general a range of $+10 \mathrm{dBm}$ to $+20 \mathrm{dBm}$ (10 to $100 \mathrm{~mW}$ ) seems to be common figures [9].

The limitations imposed by the available WiFi transmission power of the mobile phones are illustrated in Figure 2. It plots the path losses according to

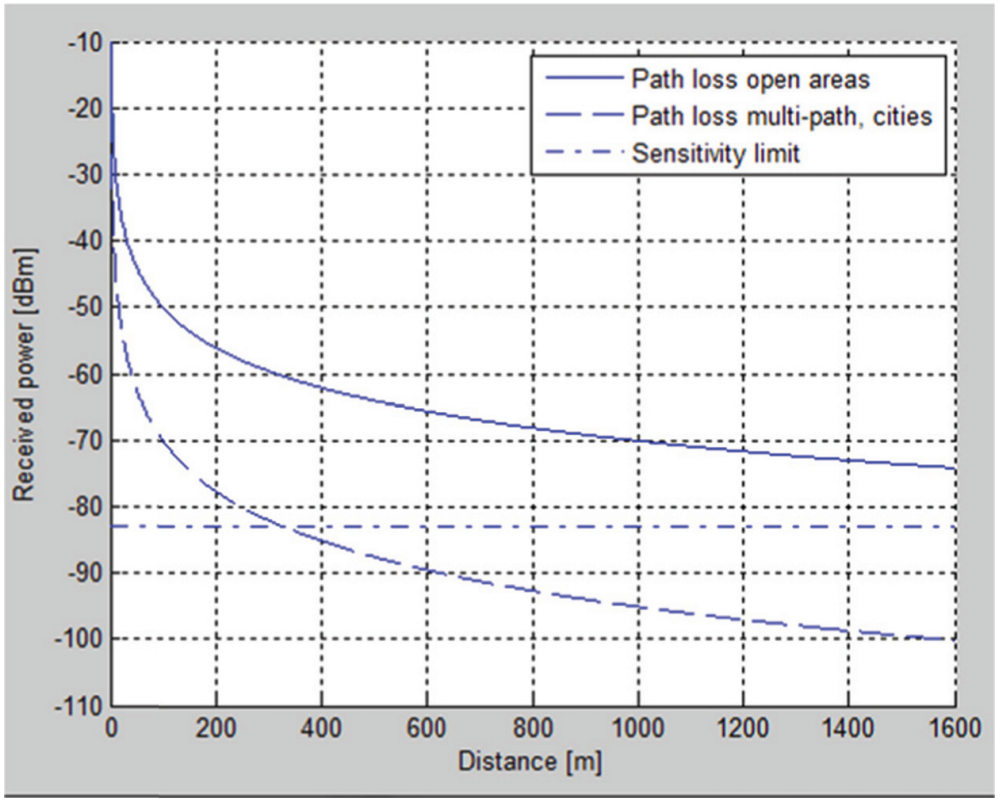

Figure 2 Transmitted power from a typical WiFi interface in a mobile phone as a function of distance. Two types of path losses are shown according to the Friis formula. Solid curve is loss in open areas (power factor is 2.0) and the dashed line is the best case loss in cities (power factor is 2.4). The dash-dot line is the sensitivity limit of a typical WiFi access point. 
Friis path-loss formula [10] as a function of distance between the transmitter and the receiver given the conditions:

- LOS

- $10 \mathrm{dBi}$ antenna gain (base station)

- WiFi frequency is $2.4 \mathrm{GHz}$

- Mobile phone transmit power is set to $100 \mathrm{~mW}$

- Bitrate is $10 \mathrm{Mbps}$

The sensitivity limit for common Wifi devices $(-84 \mathrm{dBm})$ [9] is plotted in Figure 2 (dashed-dot curve) together with the path loss for open areas (formula power is 2) and the best case path loss for cities (formula power is 2.5) [11]. For the rural villages in Africa, the true power must be between these limits, because effects such as reflections, fading, scattering, and shielding from the closely placed houses are present. Focusing at the limits where the path losses reach the sensitivity limit, it shows that the expected range is limited to approximately 300 meters in worst case and in best case the transmission power margin drops to approximately $10 \mathrm{~dB}$ at a distance of $1.6 \mathrm{~km}$. This means that under the condition described earlier the WiFi transmitter in the mobile phone will be able to connect to the WiFi base-station, if the range is below 300 meters. In the range from 300 meters up till 1600 meters the connection quality depends on the environments as stated earlier. It is noted that the transmitter in the WiFi base-station will be able to reach the mobile phone, because it uses far more power compared to the mobile phone transmitter and its receiver have approximately the same sensitivity.

As discussed, communication can be established under some conditions; however, the condition of LOS cannot always be fulfilled, e.g. inside the houses where the walls will shade the base-stations and impose attenuation on the signals.

\subsection{Wall Attenuation}

Many factors have impact on the total path attenuation, where the major factors are the path loss, the transmit power, the gain of the antennas, and the wall attenuation. Most of these factors cannot easily be modified. The path loss is bounded by the laws of physics and the mobile phone transmit power (including antenna gain) is limited by battery-time and government regulations. However, the wall attenuation, which presents a considerable amount of attenuation (Table 1), can be modified. As an example, lowering the wall attenuation with e.g. $3 \mathrm{~dB}$ means the total path can be extended with approximately 100 meters (Figure 2). Hence, even light building-materials 
Table 1 The estimated wall losses (in $\mathrm{dB}$ ) as a function of electrical parameters for building materials.

\begin{tabular}{lccc}
\hline & Permittivity & Conductivity $[\mathrm{S} / \mathrm{m}]$ & Wall Loss [dB] \\
\hline Semi dry clay & 10 & 0.02 & 6.3 \\
Semi dry sand & 10 & 0.01 & 5.8 \\
Brick wall & 4.5 & 0.02 & 4.2 \\
Reinforced Concrete & 7 & 0.1 & 12.0 \\
Wood (wet) & 6 & 0.01 & 3.9 \\
\hline
\end{tabular}

like wood contribute considerably to the total path loss (attenuation of $3.9 \mathrm{~dB}$, Table 1).

A wide range of building materials are used to construct homes in developing countries like Africa. Common examples are stick/wood, mudbricks, mud, rammed earth, and stones. These materials have influence on a penetrating radio-wave, by reflecting part of it and converting part of it into heat, i.e. these materials have complex reflection and transmission coefficients.

A model of these walls can be derived by modeling them as an equally thick homogenous plate, which is characterized by the material permeability $(\mu)$, the material permittivity $(\varepsilon)$, and the material conductivity $(\sigma)$. The transmission and reflection-coefficients for this type of models is given in [12].

$$
T_{E}=\frac{\left(1-R^{2}\right) P_{d} P_{b}}{1-R^{2} P_{d}^{2} P_{a}} \quad R=\frac{\cos \theta_{i}-\sqrt{\varepsilon_{r}-\sin ^{2} \theta_{i}}}{\cos \theta_{i}+\sqrt{\varepsilon_{r}-\sin ^{2} \theta_{i}}}
$$

Where

- $P d$ is $\exp \left(-\mathrm{jk} \mathrm{k}^{\prime} \mathrm{l}\right)$

- $\mathrm{Pb}$ is $\exp (\mathrm{jkb})$

- $P a$ is $\exp (j k 21 \sin \theta t \sin \theta i)$

- $\mathrm{k}, \mathrm{k}^{\prime}$ is propagation constants in fee space and wall respectively

- $\theta \mathrm{t}, \theta \mathrm{i}, 1, \mathrm{~b}$ is defined in [12].

Similarly, the E-field part that passes through the wall will be exposed to attenuation and phase-shift according to:

$$
\begin{aligned}
& E(z)=E(0)+e^{-\alpha z} e^{-j \beta z} \\
& \alpha \cong \frac{\sigma}{2} \sqrt{\frac{\mu}{\varepsilon}} \quad \beta \cong \omega \sqrt{\mu \varepsilon}
\end{aligned}
$$

Where $\mathrm{E}(\mathrm{z})$ is the wave $\mathrm{E}$-field component in distance $\mathrm{z}$ from the source $\mathrm{E}(0)$. The first exponential term is the wall attenuation (energy converted into heat) and the second term is the phase shift that the wall imposes on the wave. 


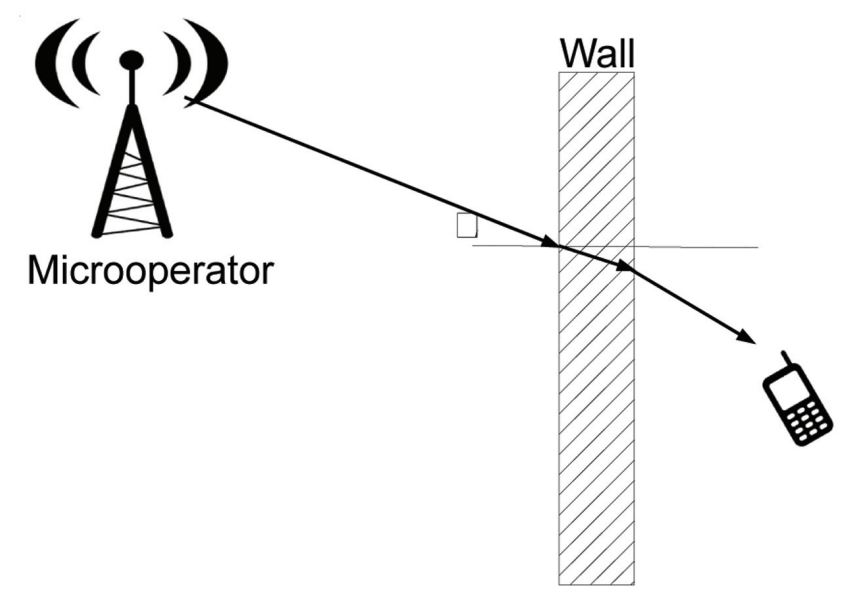

Figure 3 A scenario where a mobile phone penetrates a wall in a given angle to connect to the WiFi base station (Micro-operator).

By using the model provided in Equation (1) it is possible to explore a scenario where a mobile phone is connected to a WiFi tower (Micro-operator) from inside a home (Figure 3).

The wall losses in Figure 3 can be estimated by using formula (1) and (2) and assuming that the wall is $15 \mathrm{~cm}$ thick, the angle $(\theta \mathrm{i})$ of the incoming wave-front is 30 degrees, and the wall is made of one of the building material listed in Table 1.

By combining these wall losses with the received power (Figure 2) it can be concluded that in worst-case (pathloss multi path cities, Figure 2) a wall loss of e.g. $6 \mathrm{~dB}$ will lower the minimum range from 300 meters to approximately 150 meters. In addition, the received power curves for open areas (Figure 2) are very flat from approximately 800 meters to 1600 meters with some margins; however, even a small amount of interferences or shading will most likely obstruct communications in this range.

\subsection{Passive Repeaters}

As discussed, a passive repeater is simply two antennas connected together by a piece of cable and a passive phase shifter, i.e. no power consumption electronics, etc. One antenna is mounted on the wall outside the home and the other is mounted on the wall inside the home. When the antenna on the outside receives a radio wave it is converted into an electric signal (a voltage) which is transferred to the phase shifter. The phase shifter changes the signal 


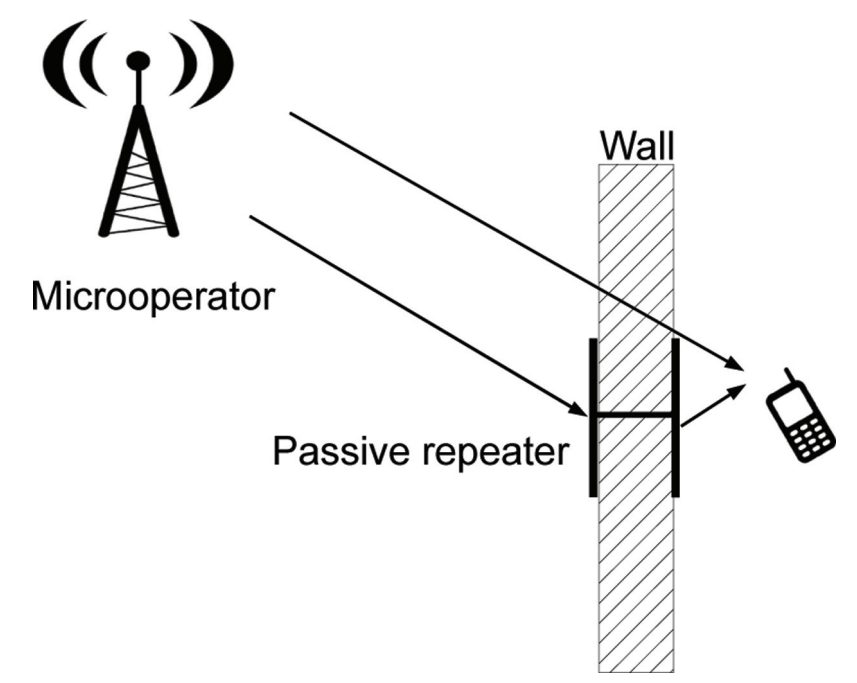

Figure 4 A scenario where a mobile phone receives a ray combined from a direct ray and a ray from the passive repeater.

phase and feeds the signal to inside antenna that retransmitted it (Figure 4). The phase shifter can in its simplest form be some pieces of cable that connects the antennas and simultaneously acting as an electrical transformer.

This concept works very well, but, it also have some disadvantages in the form of costs of the antennas and it creates an interference pattern inside the home. Firstly, the antenna costs are a onetime expense and can be low by using micro-strip based antennas. Secondly, the interference pattern is created by superposition of the direct signal penetrating the wall and the signal transmitted from the passive repeater. Each of these signals experiences a phase and an amplitude shift why they combine constructively or destructively. Hence, it is expected that there will be spots inside the home where the signal are very good and other spots where it is poor.

\subsection{A Passive Repeater Model}

The passive repeater model simplifies the calculations by approximating the true distributed radio waves with two casted rays. One beam is passing through the wall and the other is received in the passive repeater and re-transmitted inside the home (Figure 5). This type of models assumes:

- No reflections from the home walls take place

- The walls are homogenous plates 


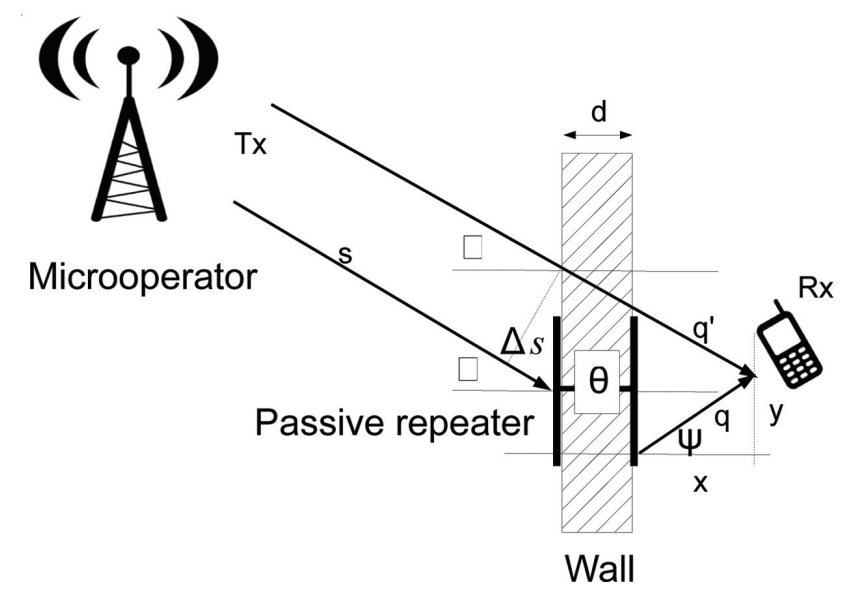

Figure 5 The model used for the passive repeater simulations.

- The walls electric parameters can be considered constant in the signal bandwidth

- The multi-reflections inside the wall are negligible.

These assumptions are valid in many of the house-types used in rural areas in Africa. In addition, the model assumes that the Micro-operator connects to the mobile phone; however, the limit is in the return path where the connection problem is from the mobile phone to the Micro-operator. This change does not make any differences, because the system is passive and reciprocal.

A model for the direct ray from the Micro-operator (Tx) to the receiver $(\mathrm{Rx})$ can be derived by using the formula for the electric far-field for a short dipole antenna A1 (annex). Combining this equation with Equation (1) yields the field transmitted through the wall (3).

$$
\bar{E}_{r d}=\frac{\sqrt{30 P_{t} G_{t}}}{s+q^{\prime}}|T| e^{-j\left(\beta\left(s+q^{\prime}\right)-\theta_{T}\right)}
$$

Where $\beta$ is the free-space phase constant, $\mathrm{r}$ is the distance, $\mathrm{Pt}$ is the transmitted power, and Gt is the transmitter antenna gain. The distance symbols in Figure 5 have been used.

Similarly, a model for the ray passing through the passive repeater path can be derived. By using the Friis formula for antennas A2 (annex) together with A1, Equation (4) can be derived.

$$
\bar{E}_{r r}=\sqrt{\frac{30 P_{t} G_{t}}{L_{c}}} \frac{\lambda}{4 \pi(s+\Delta s) q} \sqrt{G_{r r} G_{r t}} F_{r r}(\phi) F_{r t}(\phi) e^{-j \beta\left(s+\Delta s+q+\sqrt{\varepsilon_{r}} d\right)}
$$


Where Lc is phase shifter and cable losses. Gr and Gt are the respective antenna gains. $\lambda$ is the wavelength. Grr and Grt are gains for the passive repeater antennas respectively. $\operatorname{Frr}(\phi)$ and $\operatorname{Frt}(\phi)$ are the directionality functions for the passive repeater antennas, which in this context $(y=0$ in Figure 5) can be ignored and set to one. The square root of epsilon relatively compensate for the wavelength of the wall.

Finally, Equations (3) and (4) can be combined and aligned by the phase shifter (providing a shift of $\theta$ degrees, see Figure 5). Assuming vertical polarization the received field yields (5):

$$
\bar{E}_{r}=\bar{E}_{r d}+\bar{E}_{r r} e^{j \theta}
$$

\subsection{Model Simulation Results}

The simulations are carried out by implementing the models derived in Equations (1) to (5) in a mathematical tool. Regarding the input parameters the following are assumed:

- Wall thickness is $15 \mathrm{~cm}$.

- Transmitter antenna gain and power are normalized to one.

- Frequency is $2.4 \mathrm{GHz}$.

- Distance from transmitter to the home is $1 \mathrm{~km}$.

- Passive repeater antenna gains are $15 \mathrm{dBi}$ for the outer antenna and $10 \mathrm{dBi}$ for indoor antenna.

- Attenuation phase shifter is set to $1.7 \mathrm{~dB}$.

- Angle between the transmitted ray and the house wall normal vector $(\phi)$ is set to 30 degrees.

Running the simulation model with these parameters on a "semi-dry clay" wall as a function of different phase tunings yields the results illustrated in Figure 6.

From Figure 6 it is noted that the received power divided by the power in the direct ray (y-axis) is positive in a distance ranging from 0 to more than 5 meters (x-axis) inside the home. Hence, in a range of e.g. half a meter from the wall a gain of approximately $4 \mathrm{~dB}$ can be achieved. This considerably lowers the impact of the wall attenuation (approximately $6 \mathrm{~dB}$ ) and thereby improves the coverage range for the $\mathrm{WiFi}$ base stations (see Figure 2). However, it is also noted that for a fixed setting of the phase shifter, e.g. 0 degrees, the received power is damped at the approximate distances of $0.7,1.7$, and 2.7 meters from the wall. Similarly, the power is amplified at the approximate distances of 0.4 , 1.2, and 2.2 meters from the wall. In theory, this means that the user has to 


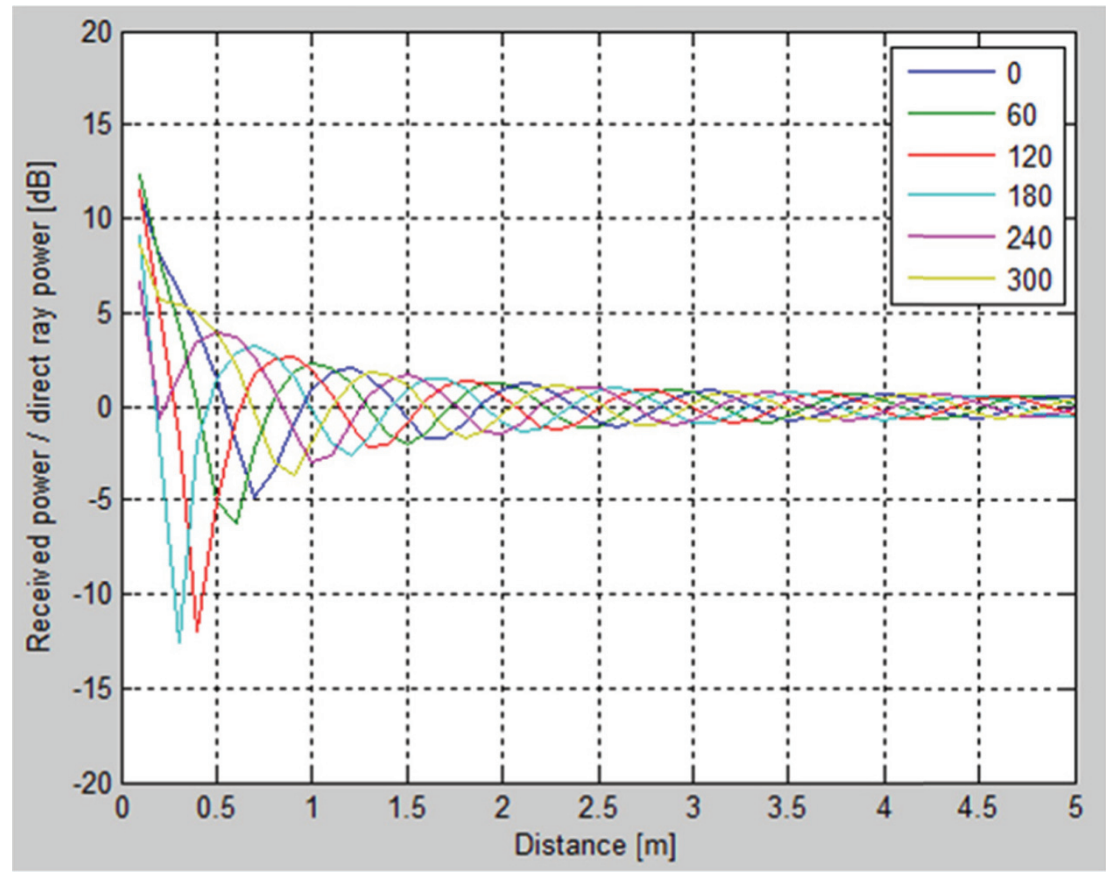

Figure 6 Simulation results for the received power relative to the received power in the direct ray as a function of distance from the wall inside the home. Each curve represents a phase angle shift provided by the phase shifter.

find optimal positions inside the home close to the amplified positions, but in the real world some reflections and scattering will occur which averages these extremes to some extent.

Another challenge is the usage of directional antennas in the passive repeater. Especially, the outdoor antenna has some gain and points towards the WiFi base station. This means that the antennas mounted at the different homes cannot communicate. This is a problem because WiFi uses a CDMA/CA based algorithm, which listens to the channel before accessing it. Thus, hiding the nodes for each other this way is known as the "hidden node problem". One possible solution is to use the RTS/CTS scheme embedded in the WiFi standard to reserve the channel and thereby avoid collisions.

The same scenario with the wall material set to "semi dry sand" and "reinforced concrete" is shown in Figure 7. As seen the impact from the "semi dry sand" loss $(5.8 \mathrm{~dB}$, Table 1$)$ can be canceled to some extent by the gain achieved by the passive repeater in a distance of half a meter $(4 \mathrm{~dB})$. 

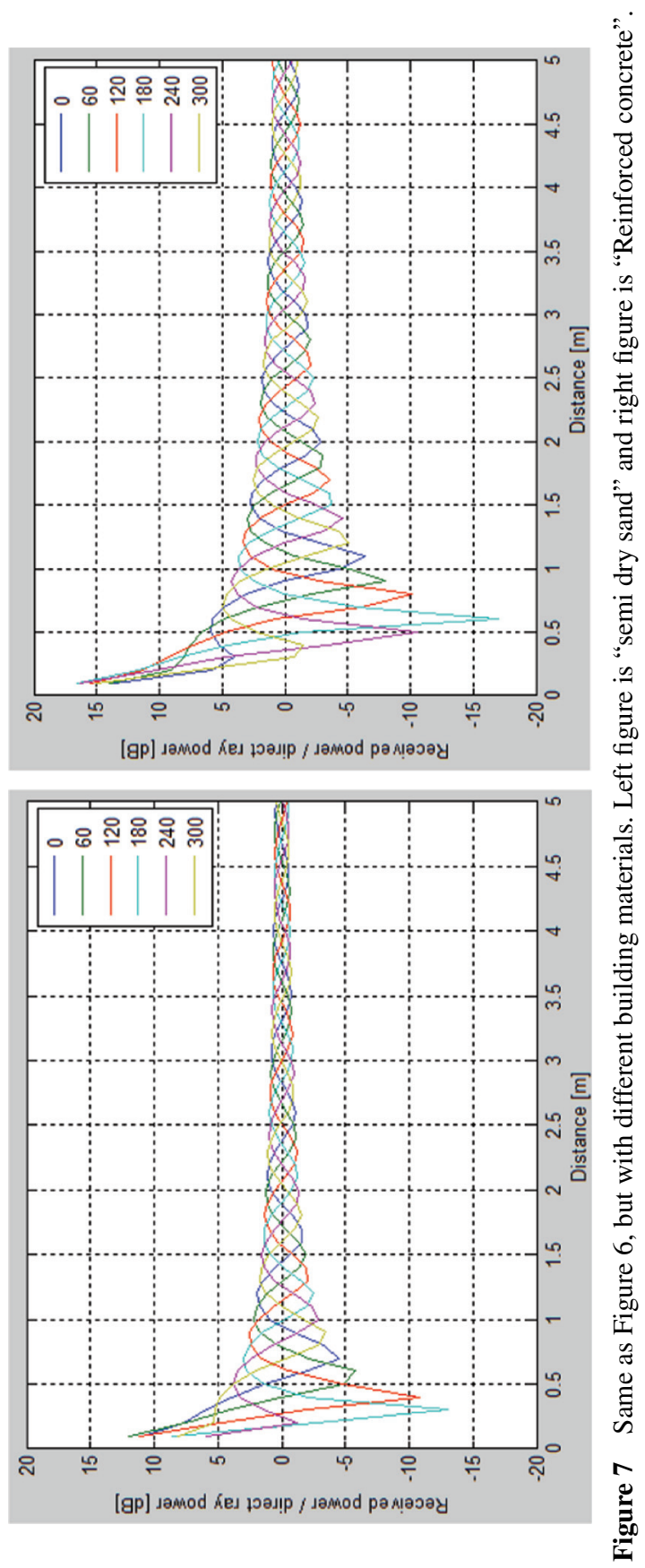
Similarly, the loss from "Reinforced concrete" $(12 \mathrm{~dB}$, Table 1$)$ can be partly canceled by the gain of the passive repeater $(7 \mathrm{~dB})$ in the distance of half a meter.

\section{Conclusion}

As stated, it is a challenge to provide indoor Internet access in the rural areas of Africa. However, using the solution provided by the company BlueTown in combination with a passive repeater overcomes some of these challenges. Thus, the main disadvantage in the BlueTown solution is the indoor WiFi coverage where the limitation is set by the available transmission-power in the mobile phones. It has been found that this range can be as low as 150 meters behind a wall build of semi dry clay (with a loss of $6.3 \mathrm{~dB}$ ). By adding a simple passive repeater the wall losses can be canceled to some extent by the gain provided $(4 \mathrm{~dB})$. This means considerable savings in the infrastructure in the form of fewer towers which can be positioned at larger distances. In addition, this solution offers benefits as it is cheap, it is sustainable, and it is power neutral, why it fits very well to rural areas in Africa as well as in other rural areas throughout the world.

From a short summary perspective, it has been found that passive repeaters are able to expand the coverage range considerably in some positions behind the walls; to some extent they simply cancel the wall losses.

Nevertheless, this solution also has disadvantages such as extending the hidden node problem, and it requires the user to be in predefined distances from the shielding wall.

\section{Annex}

Formula for the electric far-field for a short dipole:

$$
\bar{E} \cong \frac{\sqrt{30 P_{t} G_{t}}}{r} e^{-j \beta r}
$$

Friis formula for antennas:

$$
\bar{E}_{r}=\bar{E}_{t} \sqrt{G_{t} G_{r}} \frac{\lambda}{4 \pi r}
$$


Where Er and Et are the fields received and transmitted respectively. Gr and Gt are the respective antenna gains. $\lambda$ is the wavelength, and $r$ is the distance between the transmitter and the receiver.

\section{References}

[1] ITU. (2011). Available at: https://www.itu.int/ITU-D/ict/facts/2011/mate rial/ICTFactsFigures2011.pdf

[2] James, J. (2010). Mechanisms of access to the Internet in rural areas of developing countries. Telematics Informatics, 27, 370-376.

[3] Matthee, K., and Mweemba, G. (2007). "Bringing Internet connectivity to rural Zambia using a collaborative approach," in International Conference on Information and Communication Technologies and Development, ICTD 2007, 1-12.

[4] Roslee, M., and Chimheno, R. (2013). "Wireless regional access networks: a wise choice for internet connectivity to rural areas of Zimbabwe," in International Conference on Information and Communication Technologies and Development, 2007. ICTD 2007, 7.

[5] Gupta S. (2015). Rural broadband access innovation: last-mile-asmanaged service. Commun Today, 1.

[6] Hristov, H., Feick, R., and Grote, W. (2001). Improving indoor signal coverage by use of through-wall passive repeaters. IEEE Antennas Propag. 2, 158-161. doi: 10.1109/APS.2001.959645

[7] FMFI. (2015). First Mile First Inch Home Page. Available at: http:// www.fmfi.org.za/wiki/index.php/First_Mile_First_Inch_Home_Page

[8] BlueTown. (2015). Available at: http://bluetown.com/company/

[9] Zaytsev, A. (2013). "RF Design for the Mobiledevices explosion," in Cisco Connect, Dubna, 36.

[10] Shaw, J. (2013). Radiometry and the Friis transmission equation. Am. J. Phys. 81, 33. doi: 10.1119/1.4755780.

[11] Sklar, B. (2002). Digital Communications, Fundamentals and Applications, 2nd Edn. New York, NY: Pearson.

[12] Burnside, W. D. (1983). High frequency scattering by a thin lossless dielectric slab. IEEE Trans Antennas Propag. 31, doi: 10.1109/ TAP.1983.1143019 


\section{Biography}

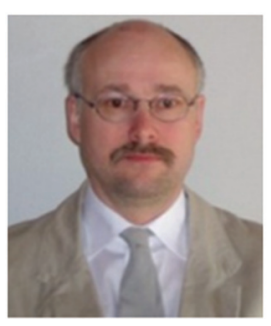

P. Lynggaard is an assistant professor in the communication, media, and information systems section at Aalborg University Copenhagen, Denmark. Dr. Lynggaard received his BSc in Electrical Engineering from Technical University of Denmark, and his MSc and Ph.D. from Aalborg University.

Dr. Lynggaard has many years' experience of industrial development within a broad range of areas, e.g., signal processing, digital electronics, software development, artificial intelligence, SDR and IoT. Through the years, he has given conference presentations, given lectures, and published papers in these and other topics at Technical University of Denmark and at Aalborg University, Denmark. 